\title{
Digital medical education empowered by intelligent fabric space
}

\author{
Min Chen ${ }^{1,2}$, Rui Wang ${ }^{1,2, \#}$, Rui Wang ${ }^{1, \#}$, Yingting Zhou ${ }^{1,2}$, Zicheng He ${ }^{1}$, Xiaojuan Liu ${ }^{1}$, Muyao He ${ }^{1,2}$, \\ Jiaxi Wang ${ }^{1,2}$, Chaolin Huang ${ }^{3}$, Huamin Zhou ${ }^{4}$, Ping Hong ${ }^{5}$, Chong Hou ${ }^{1,6}$, Ning Zhou ${ }^{1,7}$, \\ Dingyu Zhang $^{8}$ \& Guangming Tao ${ }^{1,4, *}$ \\ ${ }^{1}$ Sport and Health Initiative, Optical Valley Laboratory and Wuhan National Laboratory for Optoelectronics, Huazhong University of Science \\ and Technology, Wuhan 430074, China; \\ ${ }^{2}$ School of Computer Science and Technology, Huazhong University of Science and Technology, Wuhan 430074, China; \\ ${ }^{3}$ Wuhan Jinyintan Hospital, Wuhan 430048, China; \\ ${ }^{4}$ State Key Laboratory of Material Processing and Die \& Mould Technology, School of Materials Science and Engineering, Huazhong \\ University of Science and Technology, Wuhan 430074, China; \\ ${ }^{5}$ Beijing Sport University, Beijing 100091, China; \\ ${ }^{6}$ School of Optics and Electronic Information, Huazhong University of Science and Technology, Wuhan 430074, China; \\ ${ }^{7}$ Tongji Medical College, Huazhong University of Science and Technology, Wuhan 430074, China; \\ ${ }^{8}$ Hubei Provincial Health and Health Committee, Wuhan 430015, China \\ \#Contributed equally to this work. \\ *Corresponding author (email: tao@hust.edu.cn)
}

Received 3 January 2022; Revised 14 January 2022; Accepted 15 January 2022; Published online 8 April 2022

\begin{abstract}
Medical education plays an important role in promoting the development of global medical science. Nevertheless, the intrinsic gap existing between institutional medical teaching and practical clinical tasks causes low education efficiency and students' weak initiative. Recent developments of sensing fabric and embedded computing, along with the advances in artificial intelligence (AI) and digital twin technology are paving the way for the transformation of medical research towards digitization. In this work, we present an intelligent fabric space based on novel functional fabric materials and digital twin networking enabled by $5 \mathrm{G}$ and internet of things (IoT) technologies. In this space, medical students can learn knowledge with collaborative mapping of the digital and real world, cyber-physical interaction and real-time tactile feedback. And the proposed service system will evaluate and feedback students' operational behaviors to improve their experimental skills. We provide four typical applications of intelligent fabric space for medical education, including medical education training, health and behavior tracking, operation playback and reproduction, as well as medical knowledge popularization. The proposed intelligent fabric space has the potential to promote innovative technologies for training cutting-edge medical students by effective and efficient ways.
\end{abstract}

Keywords: medical education, intelligent fabric, digital twin, tactile interaction

Medical education aims to cultivate the skills and expertise of medical students, spread medical knowledge and promote the development of global medical science [1,2]. However, it is a challenge to solidify medical knowledge dissemination due to its practicality and complexity.

Advances in computing and communication technologies, such as artificial intelligence (AI), digital twin

(C) The Author(s) 2022. Published by China Science Publishing \& Media Ltd. and EDP Sciences. This is an Open Access article distributed under the terms of the Creative Commons Attribution License (https://creativecommons.org/licenses/by/4.0), which permits unrestricted use, distribution, and reproduction in any medium, provided the original work is properly cited. 
networking, 5G and internet of things (IoT) are enabling the design and development of next generation digital medical education. In the past few years, much of the medical research has focused on issues related to various aspects in the clinical setting, such as patient monitoring and healthcare, personalized medical treatment and robot surgery [3,4]. At present, the following shortcomings are observed during the training of medical students:

(1) Offline knowledge acquisition, examination, diagnosis and management. Currently, the medical knowledge acquisition mainly relies on offline efforts, e.g., reading papers and watching recorded videos, which is difficult to be integrated with practical scenarios. Such situation incurs with the sharp shortage of medical training resources, since online medical resources usually are not conducive to the training of doctors.

(2) Deficiency of on-spot medical operation standardization. The situations faced by doctors in the clinic are complex and variable. Accreditation Council for Graduate Medical Education (ACGME) is a representative standard for the existing medical education system in the clinical practice [5]. However, setting a standard to evaluate doctors in non-clinical exercise is difficult.

(3) Coarse evaluation through video recording without multiple physical parameters retrieval, e.g., detailed tactile interaction during operation. It is difficult to implement a cooperative perception of multiple physical quantities. Thus, for medical education, there is currently a lack of multimodal sensing and visualization equipment for the monitoring and feedback of the physiological status of doctors and patients during operation.

After decades of research, various electronic products are gradually exhibiting the features of miniaturization, softness and wearable intelligence [6-11]. Meanwhile, recent advances in fiber technologies have produced a variety of special fiber structures and functions, such as biosensors [12], supercapacitors [13,14], batteries [15-17], piezo or triboelectric generators [18], solar cells [19], light-emitting electrochemical cells [20] and light-emitting devices [21,22], etc. Intelligent fabric sensors [23] have also been applied to monitor motions and external stimuli, especially in the surgical or micro-nano manipulation fields. And it is critical to achieve accurate motion detection and tactile sensing [24-28]. Gloves based on the stretchable and flexible sensors have been recently investigated [29-33]. In our work, a variety of perceptions of physical quantities (sound, light, electricity, heat, magnetism, force, etc.) are realized through intelligent fabric. The metrics and regulation of these physical quantities are the foundation of digitized intelligence. The progress in the field of materials science provides strong support for the construction of medical education platforms.

Digital twin involves the establishment of a spatiotemporal, multidimensional, multi-disciplinary and dynamic model for mapping the physical entities of real space in a digital way, to simulate the attributes, behaviors and rules of such physical entities [34,35]. It can be used to describe complicated conditions in medical scenarios and the fusion state between the physical world and information space. The status of medical personnel and environment is first reflected on the simulation space. Then, the feasible actions in the virtual world are fed back to the physical world [36]. The potential real-time skill evaluation technologies with low latency and high interoperability for future medical education are explored in this article.

Figure 1 shows an intelligent fabric space created with digital twin technology. Such space brings digitization into medical education. In the intelligent medical space, fabrics with detection, simulation, and interactive functions allow medical students to experience immersive operations. It is a digital method to monitor the behaviors and states of both patients and doctors and standardize the practice of medical students 


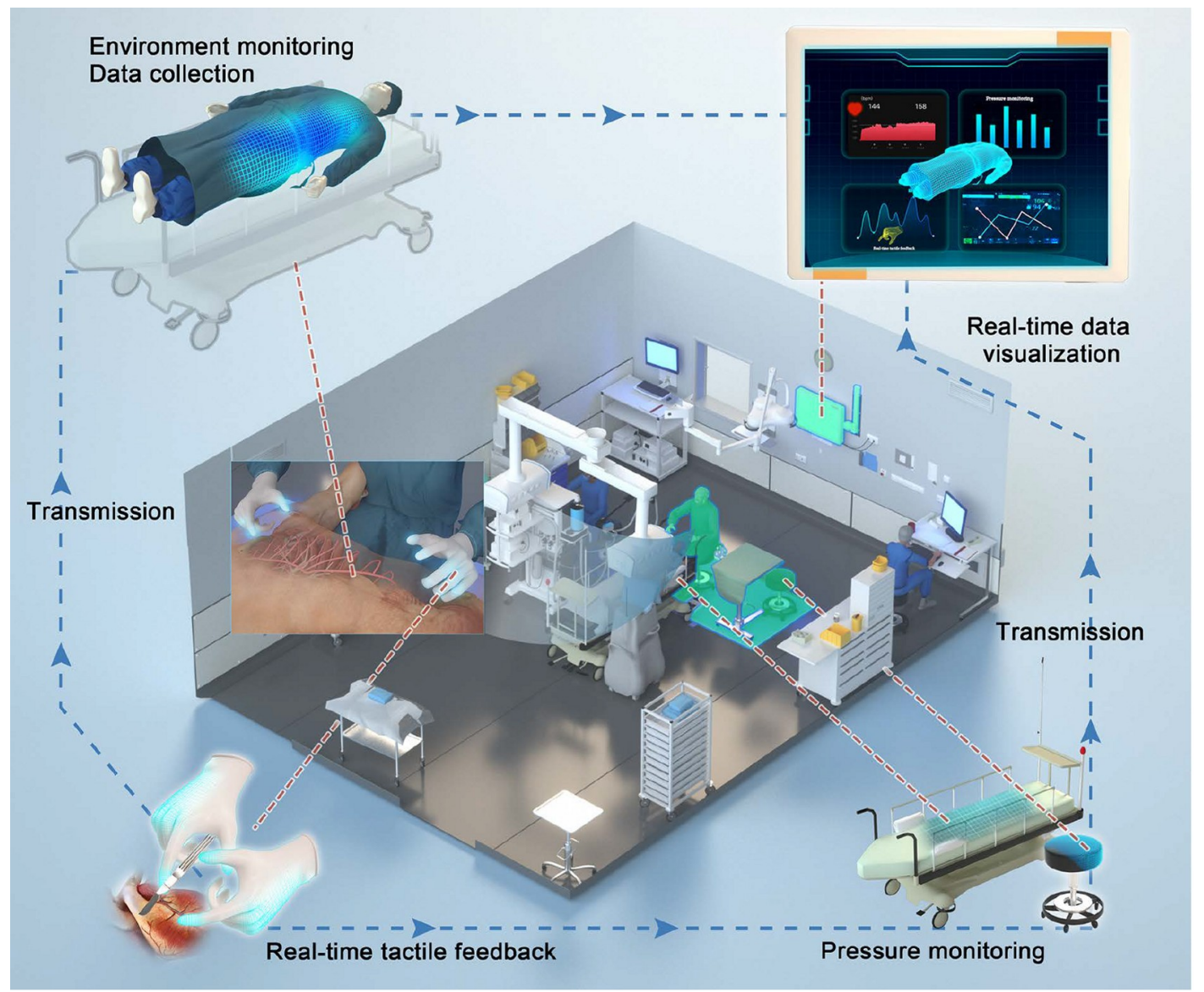

Figure 1 Digital medical education empowered by intelligent fabric space. An intelligent fabric space is created with collaborative mapping of the digital and real world, cyber-physical world interaction and real-time tactile feedback for medical education.

through simulation and evaluation, improving the quality of medical education and ensuring the smooth completion of surgery practice.

\section{Intelligent fabrics enabled digital medical education}

The advantages of non-inductive and multi-extensible fabrics with high sensitivity can address the problems of data collection and operational action evaluation in the field of medical education. When 5G networks and AI meet sensing fabrics, a comprehensive network infrastructure with powerful processing capabilities can be provided for medical education scenarios [35,37]. The architecture of intelligent fabric space is presented in this section.

\section{Architecture of intelligent fabric space}

To achieve digitization of medical education in an intelligent fabric space, the actions and behaviors of the executor first need to be collected, evaluated and simulated. Figure 2 shows the system architecture of the 
intelligent fabric space. When integrated with $6 \mathrm{G}$ intelligence networking technology [38], intelligent fabric space can renovate a wide range of future applications in terms of smart cities, smart living, medical care and education.

As shown in Figure 2, the first layer is fabric terminal layer, including a variety of intelligent fabric sensor nodes. In general, flexible fabric nodes are characterized by strong tensile performance, high sensitivity and low responsive delay. In contrast to conventional sensory devices, fabric sensors can be embedded into any clothing, furniture, dummy body and other flexible items to collect multi-source data in a nearly imperceptible way. The different kinds of fabric materials include piezoelectric [39-42], triboelectric $[33,43,44]$, magnetoelectric [45,46], temperature transducers [47], optical sensor [48] and capacitance sensors [49]. They exhibit great potential in environmental perception, human health monitoring and behavior recognition. In the future, invisible fabric nodes will become a novel way for interaction between humans and computers in daily life.

The second layer is network transmission layer. It is responsible for the low-latency and high-reliability communication for massive data [50]. In 5G networks, some challenges, such as the highly dynamic changes of the transmission link, will be handled for providing real-time connection services. Efficient intercommunication among humans, machines and objects is established in this layer to improve perception accuracy with various distances [51]. In order to meet the requirements of distributed fabric signal acquisition and algorithm analysis, the network transmission layer ensures the reliable sensing of the first layer and the timely feedback of computation result in the third layer.

The third layer is edge cloud layer which has two major tasks, i.e., real-time data analysis and situation recognition for diverse scenes. Typically, terminal nodes lack computing power with limited local storage, and hard-to-meet quality-of-service requirements in terms of delay and accuracy. For tasks with low latency tolerance, lightweight models are deployed on the edge server and the calculated results are fed back to the

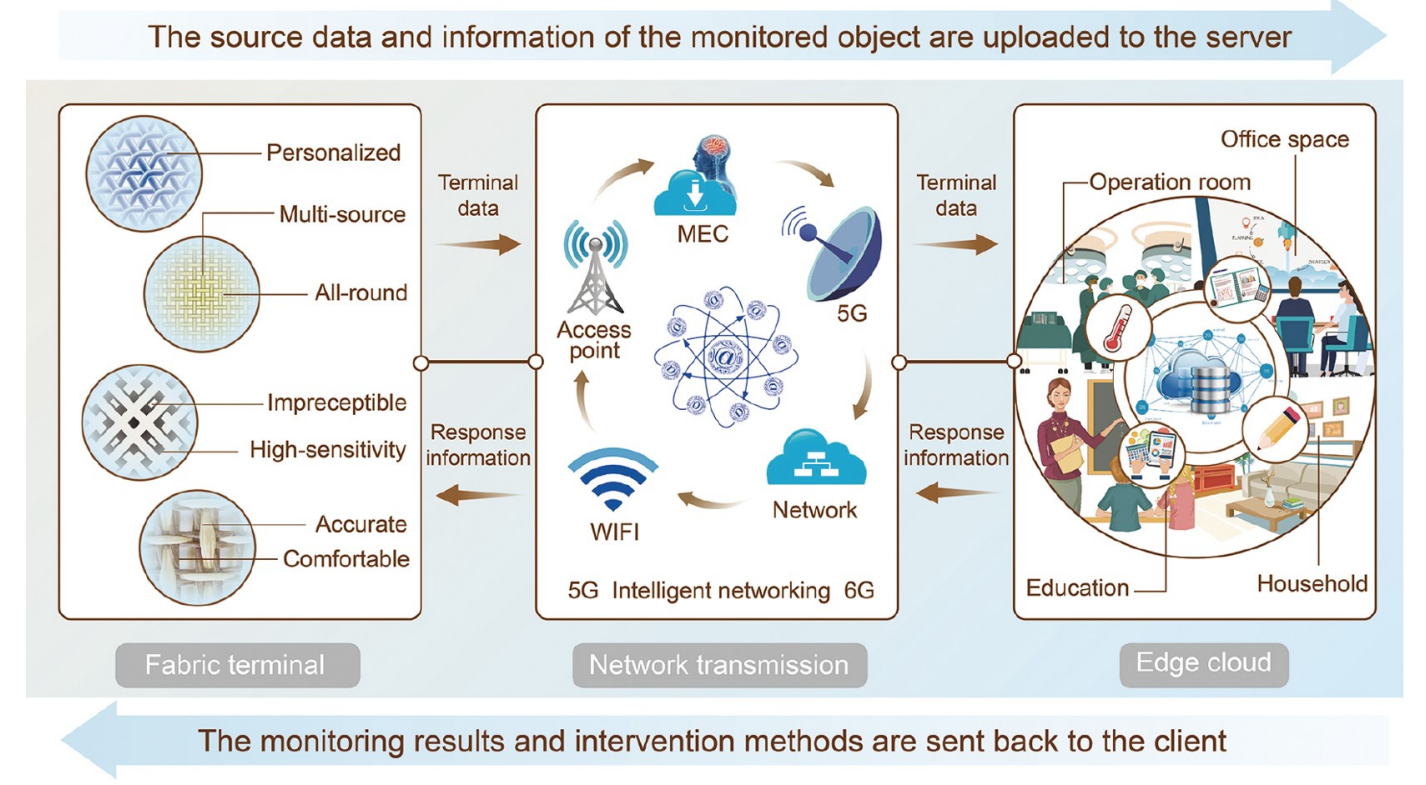

Figure 2 System architecture of the intelligent fabric space includes fabric terminal layer, network transmission layer and edge cloud layer. The first layer is responsible to perceive information from object and environment. The second layer transmits data to the first layer and the third layer. And the third layer deploys AI algorithms to process data on server. 
terminal nodes. Tasks requiring high computing power are processed on the cloud with the high performance server [52]. The advances in graphic processing unit and the development of deep learning have significantly enhanced the processing capabilities and expanded the analysis methods for multi-modal data in many fields, which also become a strong support for the analysis of large-scale fabric sensing data [53].

\section{Medical education driven by digital twins}

Based on the architecture of intelligent fabric space, a variety of application scenarios can be designed for medical education. To provide an adequate and vivid clinical operating environment, the digital twin technology is employed to establish the virtual experimental platform by combining the physical world and virtual space.

We use different fabric materials to construct the medical education environment, which possesses the features of high-resolution, high-sensitivity and non-contact sensing. In this section, a "four-space" model is proposed, which includes physical space, virtual space, service system and service interface [54]. Then, we build a digital twin platform based on the "four-space" model for medical education, as shown in Figure 3.

(1) Physical space refers to the objective entity that exists in the physical world. In the medical education scene, the physical space includes the operation room, interactive environment, intelligent dummy body and fabric sensors, such as fabric medical gloves and fabric surgical gowns. Compared with the traditional one, our proposed intelligent dummy body deploys sensing fabric sensors on its skin and organs, for enabling realtime tactile feedback during medical education.

(2) Virtual space refers to a mirror of a physical space with a set of interaction rules among the physical objects [55]. The mannequin, medical ontology and user behavior are all digitized to establish a virtual space with evaluation and prediction abilities.

(3) Service system is a backend system that integrates data storage, AI modeling, data analytics, resource optimization, data visualization, and education performance evaluation. In data visualization subsystem, the operation process can be simulated based on data obtained from fabric sensors. Such process can be visualized on screen, as shown in Figure 3. In the education performance evaluation subsystem, the operation skills of medical students are evaluated based on various fabric sensory data and the detailed tactile interaction information during operation, in order to improve their operation skills.

(4) Service interface includes the interaction interfaces among physical space, virtual space and service system. It constantly updates the interactive information with the arrivals of real-time data. Service interface provides the personalized access of operation, evaluation and visualization in the physical space and virtual space.

Through "human-machine-fabric" connection [56-58], the user in the digitized medical education space can receive an immersive operation experience with ultra-low latency and high-fidelity, via the abovementioned four spaces.

\section{Typical applications of intelligent fabric space-empowered medical education}

Based on advances of intelligent fabric space and digital twin system, we present four typical applications in 


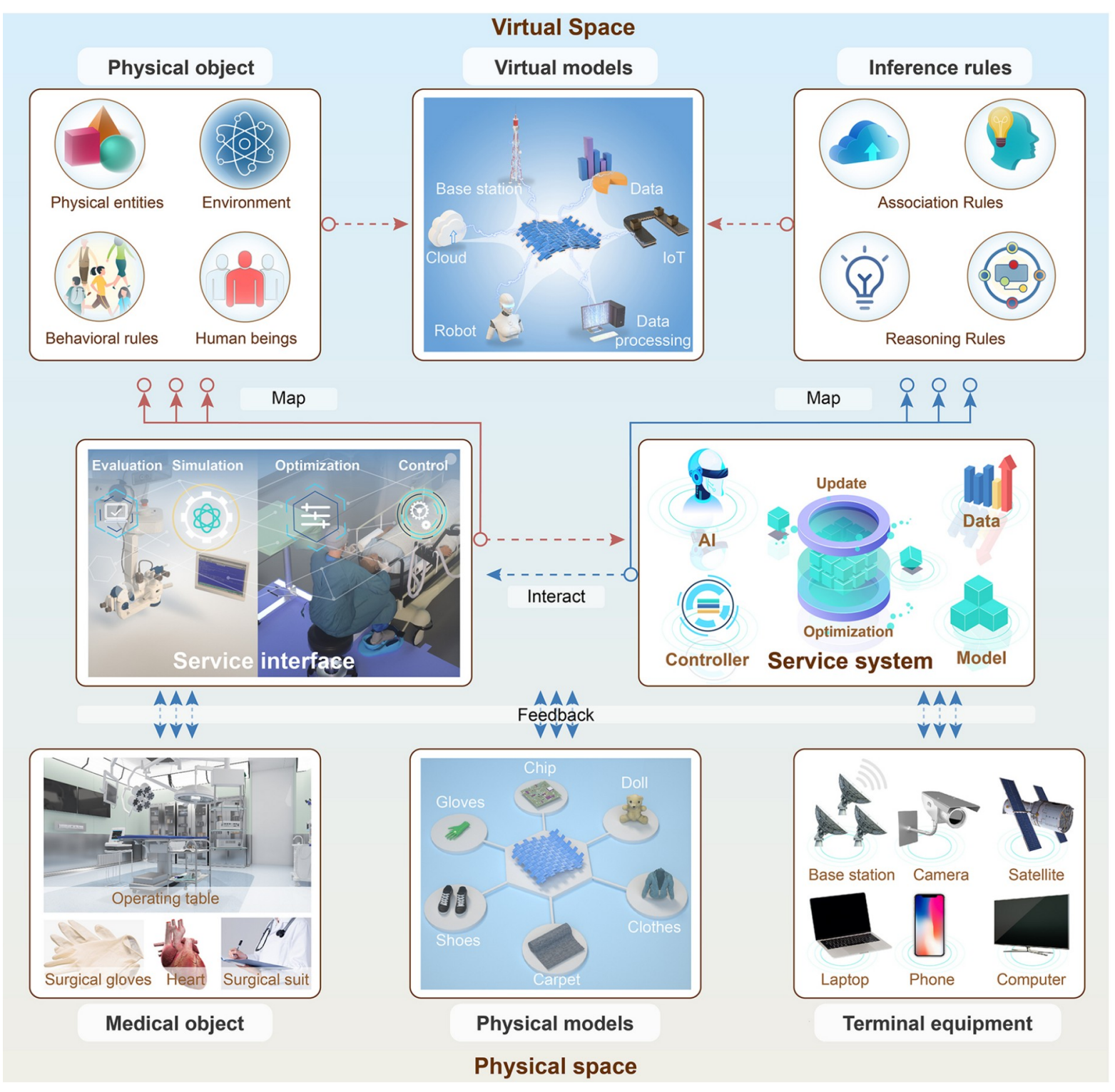

Figure 3 The interactive framework from physical to virtual world, driven by digital twin including four modules. Physical space is the physical world. Virtual space is the simulation world. Service system provides operation functions for executors. Service interface is the interactive interface between physical space and virtual space.

the field of medical education as shown in Figure 4.

(1) Medical education training. Medical students can perform practical exercises to expand their human anatomy knowledge more intuitively through three-dimensional visual interfaces in the intelligent space. During experiments, the system can automatically identify the posture of a student holding an instrument, record operation details and present standard evaluation results for the operating student.

(2) Health and behavior tracking. Fabric nodes embedded in protective clothing can monitor the physical condition of doctors with indicators such as temperature, heart rate and degree of concentration, and their behavioral information such as gait, sitting position and the movement trajectories. It will be used to monitor body situations to perform comprehensive operation evaluation when medical students receive medical 


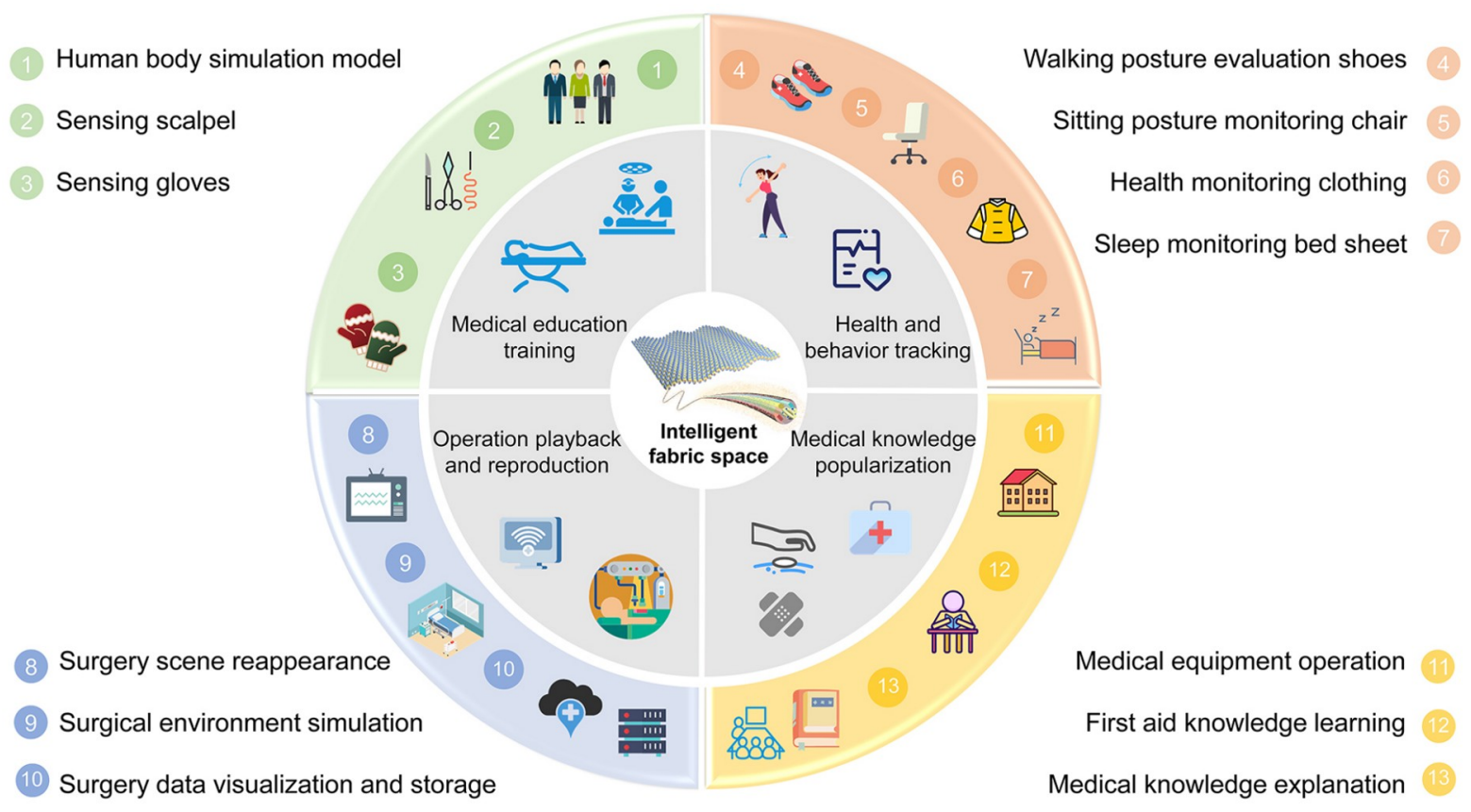

Figure 4 Four typical applications in the field of medical education. The platform supports the four typical scenarios, including medical education training, health and behavior tracking, operation playback and reproduction and medical knowledge popularization.

training.

(3) Operation playback and reproduction. The digital twin system can simulate the detailed actions and scenes of the operation to reproduce the surgical scene for both typical and rare cases by means of the recorded data collected by smart gloves and gauze during the operation. This provides doctors with an opportunity to communicate before and after the surgery, solving the problem of insufficient samples in medical learning.

(4) Medical knowledge popularization. The general public can learn first aid skills in an intelligent fabric medical space, by wearing intelligent fabric equipment for remote learning and practice independently by watching the supporting demonstration operation process. In this way, first aid education will be extended to every household in a low-cost but efficient way.

The digital twin system with intelligent fabrics as the core can nullify the limitations of time and space, allowing experts, medical students and the public to obtain appropriate medical knowledge to support healthy lives.

\section{Outlook and perspectives}

Important breakthroughs have been achieved in high-performance fabric materials with flexible tensile strength, strong sensing and high degree of plasticity. Intelligent fabric can be used to realize collaborative perception of multiple physical quantities, with the metrics and regulation of these physical quantities as the foundation of digitized intelligence. The widespread deployment of $5 \mathrm{G}$ networks and the perspective of $6 \mathrm{G}$ 
theory have promoted the development of functional fibers, as well as embedded intelligent fabric in the field of medical education. In this article, intelligent fabric and digital twin are combined to create a cyber-physical system of medical scenarios to serve medical students. Based on the architecture of intelligent fabric space, the simulation of medical education scene and the corresponding interactions driven by digital twin technology is realized.

However, the scheme proposed in this work still has limitations in terms of ubiquitous fabric sensing and distributed algorithm processing. The perceiving performance of some fabrics is not stable, which causes difficulty for large-scale deployment. Meanwhile, although the advanced network architecture provides realtime data transmission, the current algorithms are still not efficient on processing spatiotemporal multidimensional sensory data when facing a large amount of computation requirements. This motivates us to constantly strive to solve problems at the aspects of perception, algorithm and deployment.

In the future, the user of medical education will be able to experience an immersive operation with ultralow latency and dynamic high-fidelity. With fabric sensing materials, the interaction between human and semi-simulated organs can be captured in detail, which also facilitates human body modeling. Driven by computer science and materials science, fabric and digital twin technology can create a virtual surgery practice environment to aid medical students to perform comprehensive training for improving education quality while saving medical resources.

\section{Funding}

This work was supported by the National Natural Science Foundation of China (62175082 and 61875064).

\section{Author contributions}

M.C., R.W., R.W., Y.Z. and G.T. designed the research and wrote the initial manuscript. Z.H., X.L., M.H. and J.W. made the literature review and realized the figures. C.H., H.Z., P.H., C.H., N.Z. and D.Z. provided the source supports and supervision. All authors have contributed to the discussion of the content and have revised and edited the manuscript.

\section{References}

1 Vitt JR, Sun CH, LeRoux PD, et al. Minimally invasive surgery for intracerebral hemorrhage. Curr Opin Crit Care 2020; 26: 129-136.

2 Schilde S, Delank KS, Arbab D, et al. Minimally invasive vs open akin osteotomy. Foot Ankle Int 2021; 42: $278-286$.

3 Abhinav G, Subrahmanyam SN. Artificial intelligence in healthcare. J Drug Deliv Ther 2019; 9: 164-166.

4 Jiang F, Jiang Y, Zhi H, et al. Artificial intelligence in healthcare: Past, present and future. Stroke Vasc Neurol 2017; 2: 230-243.

5 Epstein RM. Assessment in medical education. $N$ Engl J Med 2007; 356: 387-396.

6 Yan W, Dong C, Xiang Y, et al. Thermally drawn advanced functional fibers: New frontier of flexible electronics. Mater Today 2020; 35: 168-194.

7 Chen M, Zhou J, Tao G, et al. Wearable affective robot. IEEE Access 2018; 6: 64766-64776.

$8 \mathrm{Hu}$ R, Song J, Liu Y, et al. Machine learning-optimized Tamm emitter for high-performance thermophotovoltaic system with detailed balance analysis. Nano Energy 2020; 72: 104687.

9 Wang R, Du Z, Xia Z, et al. Magnetoelectrical clothing generator for high-performance transduction from biomechanical energy to electricity. Adv Funct Mater 2022; 32: 2107682.

10 Zeng S, Pian S, Su M, et al. Hierarchical-morphology metafabric for scalable passive daytime radiative cooling. Science 2021; 373: 692-696. 
11 Ma Y, Ouyang J, Raza T, et al. Flexible all-textile dual tactile-tension sensors for monitoring athletic motion during Taekwondo. Nano Energy 2021; 85: 105941.

12 Liu L, Zhao F, Liu W, et al. An electrochemical biosensor with dual signal outputs: Toward simultaneous quantification of $\mathrm{pH}$ and $\mathrm{O}_{2}$ in the brain upon ischemia and in a tumor during cancer starvation therapy. Angew Chem Int Ed 2017; 56: 10471-10475.

$13 \mathrm{Ma} \mathrm{W}$, Chen $\mathrm{S}$, Yang $\mathrm{S}$, et al. Hierarchical $\mathrm{MnO}_{2}$ nanowire/graphene hybrid fibers with excellent electrochemical performance for flexible solid-state supercapacitors. J Power Sources 2016; 306: 481-488.

14 Li P, Jin Z, Peng L, et al. Stretchable all-gel-state fiber-shaped supercapacitors enabled by macromolecularly interconnected 3D graphene/nanostructured conductive polymer hydrogels. Adv Mater 2018; 30: 1800124.

15 Ren J, Zhang Y, Bai W, et al. Elastic and wearable wire-shaped lithium-ion battery with high electrochemical performance. Angew Chem Int Ed 2014; 53: 7864-7869.

16 Ren J, Li L, Chen C, et al. Twisting carbon nanotube fibers for both wire-shaped micro-supercapacitor and microbattery. Adv Mater 2013; 25: 1155-1159.

17 He J, Lu C, Jiang H, et al. Scalable production of high-performing woven lithium-ion fibre batteries. Nature 2021; 597: $57-63$.

18 Li X, Lin ZH, Cheng G, et al. 3D fiber-based hybrid nanogenerator for energy harvesting and as a self-powered pressure sensor. ACS Nano 2014; 8: 10674-10681.

19 Fu X, Sun H, Xie S, et al. A fiber-shaped solar cell showing a record power conversion efficiency of $10 \%$. J Mater Chem A 2018; 6: 45-51.

20 Pei Q, Yu G, Zhang C, et al. Polymer light-emitting electrochemical cells. Science 1995; 269: 1086-1088.

21 Zheng H, Zhang Z, Jiang S, et al. A shape-memory and spiral light-emitting device for precise multisite stimulation of nerve bundles. Nat Commun 2019; 10: 2790.

22 Shi X, Zuo Y, Zhai P, et al. Large-area display textiles integrated with functional systems. Nature 2021; 591: 240-245.

23 Shi J, Liu S, Zhang L, et al. Smart textile-integrated microelectronic systems for wearable applications. Adv Mater 2020; 32: 1901958.

24 Bandari N, Dargahi J, Packirisamy M. Tactile sensors for minimally invasive surgery: A review of the state-of-the-art, applications, and perspectives. IEEE Access 2020; 8: 7682-7708.

25 Dong B, Shi Q, He T, et al. Wearable triboelectric/aluminum nitride nano-energy-nano-system with self-sustainable photonic modulation and continuous force sensing. Adv Sci 2020; 7: 1903636.

26 Gul JZ, Sajid M, Choi KH. Retracted Article: 3D printed highly flexible strain sensor based on TPU-graphene composite for feedback from high speed robotic applications. J Mater Chem C 2019; 7: 4692-4701.

27 Fujiwara E, Santos MFM, Suzuki CK. Flexible optical fiber bending transducer for application in glove-based sensors. IEEE Sens J 2014; 14: 3631-3636.

28 Dahroug B, Tamadazte B, Weber S, et al. Review on otological robotic systems: toward microrobot-assisted cholesteatoma surgery. IEEE Rev Biomed Eng 2018; 11: 125-142.

29 Hammond F L, Menguc Y, Wood R J. Toward a modular soft sensor-embedded glove for human hand motion and tactile pressure measurement. In: IEEE/RSJ International Conference on Intelligent Robots and Systems, Chicago, 2014. 4000-7.

30 Dong B, Yang Y, Shi Q, et al. Wearable triboelectric-human-machine interface (THMI) using robust nanophotonic readout. ACS Nano 2020; 14: 8915-8930.

31 Wang W, Yu A, Liu X, et al. Large-scale fabrication of robust textile triboelectric nanogenerators. Nano Energy 2020; 71: 104605 .

32 Wang Y, Wu H, Xu L, et al. Hierarchically patterned self-powered sensors for multifunctional tactile sensing. Sci Adv 2020; 6: eabb9083.

33 Jin T, Sun Z, Li L, et al. Triboelectric nanogenerator sensors for soft robotics aiming at digital twin applications. Nat Commun 2020; 11: 5381.

34 Tao F, Cheng J, Qi Q, et al. Digital twin-driven product design, manufacturing and service with big data. Int $J A d v$ 
Manuf Technol 2018; 94: 3563-3576.

35 Tao F, Zhang H, Liu A, et al. Digital twin in industry: State-of-the-art. IEEE Trans Ind Inf 2019; 15: $2405-2415$.

36 Erol T, Mendi A F, Dogan D. The Digital Twin Revolution in Healthcare. In: 2020 4th International Symposium on Multidisciplinary Studies and Innovative Technologies (ISMSIT), Turkey: IEEE, 2020, 1-7.

37 Li Q, Xia B, Huang H, et al. TRAC: Traceable and revocable access control scheme for mHealth in 5G-enabled IioT. IEEE Trans Ind Inf 2022; 18: 3437-3448.

38 Hao Y, Miao Y, Chen M, et al. 6G cognitive information theory: A mailbox perspective. Big Data Cogn Comput 2021; 5: 56 .

39 Liu H, Wu H, Ong KP, et al. Giant piezoelectricity in oxide thin films with nanopillar structure. Science 2020; 369: 292297.

40 Qin Y, Wang X, Wang ZL. Microfibre-nanowire hybrid structure for energy scavenging. Nature 2008; 451: 809-813.

41 Han M, Wang H, Yang Y, et al. Three-dimensional piezoelectric polymer microsystems for vibrational energy harvesting, robotic interfaces and biomedical implants. Nat Elect 2019; 2: 26-35.

42 Sundaram S, Kellnhofer P, Li Y, et al. Learning the signatures of the human grasp using a scalable tactile glove. Nature 2019; 569: 698-702.

43 Tang Y, Zhou H, Sun X, et al. Triboelectric touch-free screen sensor for noncontact gesture recognizing. Adv Funct Mater 2020; 30: 1907893.

44 Chen B, Tang W, Wang ZL. Advanced 3D printing-based triboelectric nanogenerator for mechanical energy harvesting and self-powered sensing. Mater Today 2021; 50: 224-238.

45 Cañón Bermúdez GS, Karnaushenko DD, Karnaushenko D, et al. Magnetosensitive e-skins with directional perception for augmented reality. Sci Adv 2018; 4: eaao2623.

46 Melzer M, Mönch JI, Makarov D, et al. Wearable magnetic field sensors for flexible electronics. Adv Mater 2015; 27: $1274-1280$.

47 Zhang L, Lin S, Hua T, et al. Fiber-based thermoelectric generators: Materials, device structures, fabrication, characterization, and applications. Adv Energy Mater 2018; 8: 1700524.

48 Bai H, Li S, Barreiros J, et al. Stretchable distributed fiber-optic sensors. Science 2020; 370: 848-852.

49 Cooper CB, Arutselvan K, Liu Y, et al. Stretchable capacitive sensors of torsion, strain, and touch using double helix liquid metal fibers. Adv Funct Mater 2017; 27: 1605630.

50 Liyanaarachchi SD, Riihonen T, Barneto CB, et al. Optimized waveforms for 5G-6G communication with sensing: Theory, simulations and experiments. IEEE Trans Wireless Commun 2021; 20: 8301-8315.

51 Fortino G, Savaglio C, Spezzano G, et al. Internet of things as system of systems: A review of methodologies, frameworks, platforms, and tools. IEEE Trans Syst Man Cybern Syst 2021; 51: 223-236.

52 Siriwardhana Y, Porambage P, Liyanage M, et al. A survey on mobile augmented reality with 5G mobile edge computing: Architectures, applications, and technical aspects. IEEE Commun Surv Tutorials 2021; 23: 1160-1192.

53 Qiu Y, Yin W, Wang L. A high-performance and scalable NVMe controller featuring hardware acceleration. IEEE Trans Comput-Aided Des Integr Circuits Syst 2021; https://doi/org/10.1109/TCAD.2021.3088784.

$54 \mathrm{Cao} \mathrm{K}, \mathrm{Hu} \mathrm{S}$, Shi Y, et al. A survey on edge and edge-cloud computing assisted cyber-physical systems. IEEE Trans Ind Inf 2021; 17: 7806-7819.

55 Chen M, Jiang Y, Guizani N, et al. Living with I-fabric: Smart living powered by intelligent fabric and deep analytics. IEEE Network 2020; 34: 156-163.

56 Ji B, Han Y, Liu S, et al. Several key technologies for 6G: Challenges and opportunities. IEEE Comm Stand Mag 2021; 5: 44-51.

57 Hao Y, Chen M, Gharavi H, et al. Deep reinforcement learning for edge service placement in softwarized industrial cyber-physical system. IEEE Trans Ind Inf 2021; 17: 5552-5561.

58 Chen M, Xiao W, Hu L, et al. Cognitive wearable robotics for autism perception enhancement. ACM Trans Internet Technol 2021; 21: 97. 